\title{
Variational description of multi-fluid hydrodynamics: Coupling to gauge fields
}

\author{
Reinhard Prix \\ Max-Planck-Institut für Gravitationsphysik, Albert-Einstein-Institut, Am Mühlenberg 1, D-14476 Golm, German腾
}

(Dated: Feb 6, 2005)

\begin{abstract}
In this work we extend our previously developed formalism of Newtonian multi-fluid hydrodynamics to allow for coupling between the fluids and the electromagnetic and gravitational field. This is achieved within the convective variational principle by using a standard minimal coupling prescription. In addition to the conservation of total energy and momentum, we derive the conservation of canonical vorticity and helicity, which generalize the corresponding conserved quantities of uncharged fluids. We discuss the application of this formalism to electrically conducting systems, magnetohydrodynamics and superconductivity. The equations of electric conductors derived here are more general than those found in the standard description of such systems, in which the effect of entrainment is overlooked, despite the fact that it will generally be present in any conducting multi-constituent system.
\end{abstract}

\section{INTRODUCTION}

In the previous paper [1], henceforth referred to as Paper I, we have shown how the convective variational principle can be used to derive the general equations of motion for a system of interacting uncharged fluids. This variational principle is also at the heart of a series of papers by Carter and Chamel 2, 3, 4] based on a fully covariant spacetime formulation of Newtonian hydrodynamics, which is formally closer to the relativistic formulation.

Here we extend our " $3+1$ " framework developed in Paper I to allow for charged fluids and their coupling to the electromagnetic and gravitational field. In Paper I we already included the gravitational field via an explicit prescription for the external force, but now this coupling is derived in a more natural way from the variational principle using the same minimal coupling procedure as for the electromagnetic field.

Due to the coupling to the electromagnetic field, the resulting theory can only be considered an "approximate" Newtonian framework, as strict Galilean invariance will be violated. While the Newtonian hydrodynamic equations are strictly invariant under Galilean transformations, the equations of electrodynamics are invariant only under Lorentz-transformations. This well known discrepancy lead to the development of relativity, and a coupling between Newtonian physics and electromagnetism is strictly speaking either inconsistent or selects a preferred frame ("ether"). As shown in [5], demanding strict Galilean invariance of electrodynamics without an ether forces one to adopt either an "electric" or "magnetic" limit of the theory, in which certain essential effects would be absent (e.g. the magnetic force between electric currents or local charge conservation). The only fully consistent approach is to work within a (locally) Lorentz-invariant relativistic framework, as used for example in [6, 7].

From a practical point of view, however, a " $3+1$ " non-relativistic formalism is often fully sufficient in terms of precision and usually more easily applicable to many problems. The aim of this paper is therefore to provide a flexible and general framework for the description of a wide variety of charged multi-constituent systems in the "nonrelativistic" regime of small velocities and low frequencies $\left(c^{-2}\right)^{1}$. We emphasize that we do not attempt to construct a strictly Galilean-invariant theory, rather this should be regarded as a truncated theory at order $\left(c^{-2}\right)$ of a Taylor expansion of the underlying fully covariant theory.

For simplicity we restrict our analysis to non-magnetic and non-polarizable fluids, such that the vacuum Maxwellequations retain their form on the macroscopic level of hydrodynamics, and the interaction between matter and fields is restricted to the minimal coupling type. The inclusion of electric and magnetic polarization is postponed to future work.

Using this variational framework, the fundamental effects of "entrainment" and "chemical coupling", inherent to multi-constituent systems (as discussed in Paper I), are automatically included in the formalism. This is a substantial improvement over the standard "orthodox" description of electrical conductivity and charged fluids in general, in which these effects are usually completely overlooked. The present framework is also more general in the sense of being readily applicable to an arbitrary number of interacting constituents and fluids.

\footnotetext{
*Electronic address: Reinhard.Prix@aei.mpg.de

1 to be quantified in the appendix
} 
The plan of this paper is as follows: in Sec. II we extend the variational principle introduced in Paper I to the case of ("separable") coupling to the electromagnetic and gravitational fields, and derive the equations of motion for this coupled system. In Sec. III we derive the conservation of charge and mass as well as of energy and momentum from the variational principle. Sec. IV is devoted to conserved quantities under transport of the fluid flow, namely the canonical vorticity and helicity, and their special relation to superconductors. In Sec. V we discuss applications of this formalism to particular physical systems. In the appendix we derive the non-relativistic form of electrodynamic equations and show their "approximate" Galilean invariance.

\section{VARIATIONAL DESCRIPTION OF MULTI-CONSTITUENT SYSTEMS}

\section{A. Kinematics}

We follow the notation introduced in Paper I, the various constituents are indexed by capital letters $X, Y, \ldots$, which run over the constituents labels. The fundamental kinematic variables are the constituent number-densities $n_{X}$ and the associated transport currents $\boldsymbol{n}_{X}$, which are related to the respective flow-velocities $\boldsymbol{v}_{X}$ as $\boldsymbol{n}_{X}=n_{X} \boldsymbol{v}_{X}$. As in Paper I, we define the relative velocities $\boldsymbol{\Delta}_{X Y}$ between fluids $X$ and $Y$ as

$$
\boldsymbol{\Delta}_{X Y} \equiv \boldsymbol{v}_{X}-\boldsymbol{v}_{Y}
$$

In addition to the masses per particle $m^{X}$, we now admit another transported quantity, namely the charge per particle $q^{X}$. The corresponding total densities and currents are therefore the mass density $\rho$ and mass current $\rho$, given by

$$
\rho=\sum n_{X} m^{X}, \quad \text { and } \quad \boldsymbol{\rho}=\sum m^{X} \boldsymbol{n}_{X}
$$

and also the charge density $\sigma$ and charge current $\boldsymbol{j}$, defined as

$$
\sigma=\sum n_{X} q^{X}, \text { and } \quad \boldsymbol{j}=\sum \boldsymbol{n}_{X} q^{X}
$$

where here and in the following $\sum$ is used to indicate the sum over repeated constituent indices, no automatic summation over constituent indices is assumed.

In addition to the dynamics of the matter-system we also want to include the coupling to the gravitational gauge field $\Phi$ and the electromagnetic gauge fields $A_{0}$ and $\boldsymbol{A}$. The corresponding gauge-invariant field quantities are the gravitational acceleration $\boldsymbol{g}$, which is

$$
\boldsymbol{g}=-\nabla \Phi
$$

and the electric and magnetic fields $\boldsymbol{E}$ and $\boldsymbol{B}$, which are

$$
\boldsymbol{E} \equiv \nabla A_{0}-\frac{1}{c} \partial_{t} \boldsymbol{A}, \quad \text { and } \quad \boldsymbol{B} \equiv \nabla \times \boldsymbol{A} .
$$

These fields are invariant under the gauge transformations

$$
\Phi^{\prime}=\Phi+\mathcal{C}(t), \quad A_{0}{ }^{\prime}=A_{0}+\frac{1}{c} \partial_{t} \psi, \quad \text { and } \quad \boldsymbol{A}^{\prime}=\boldsymbol{A}+\nabla \psi
$$

\section{B. Dynamics}

The dynamics of the system is described by an action $\mathcal{I}$ of the form

$$
\mathcal{I}=\int \Lambda d V d t
$$

in terms of the (generally gauge-dependent) total Lagrangian $\Lambda$. Using the standard minimal coupling prescription, and assuming the electrodynamic field-contribution to be fully separable from the hydrodynamic Lagrangian, we can postulate the form of $\Lambda$ to be

$$
\Lambda=\Lambda_{\mathrm{H}}\left(n_{X}, \boldsymbol{n}_{X}\right)+\Lambda_{\mathrm{EM}}(\boldsymbol{E}, \boldsymbol{B})+\Lambda_{\mathrm{grav}}(\boldsymbol{g})+\left(\sigma A_{0}+\frac{1}{c} \boldsymbol{j} \cdot \boldsymbol{A}\right)-\rho \Phi .
$$


The gravitational field Lagrangian $\Lambda_{\text {grav }}$ has the well-known expression

$$
\Lambda_{\text {grav }}=-\frac{1}{8 \pi G}(\nabla \Phi)^{2}
$$

where $G$ is Newton's gravitational constant. The electromagnetic field contribution (cf. appendix) in the nonpolarizable case is given by

$$
\Lambda_{\mathrm{EM}}=\frac{1}{8 \pi}\left(\boldsymbol{E}^{2}-\boldsymbol{B}^{2}\right)
$$

As we have seen in the case of uncharged fluids (cf. Paper I), the hydrodynamic Lagrangian $\Lambda_{\mathrm{H}}$ defines the $d y n a m i c a l$ momenta $p_{0}^{X}$ and $\boldsymbol{p}^{X}$ per fluid particle as

$$
d \Lambda_{\mathrm{H}}=\sum\left(p_{0}^{X} d n_{X}+\boldsymbol{p}^{X} \cdot d \boldsymbol{n}_{X}\right), \quad \text { so } \quad p_{0}^{X}=\frac{\partial \Lambda_{\mathrm{H}}}{\partial n_{X}}, \quad \boldsymbol{p}^{X}=\frac{\partial \Lambda_{\mathrm{H}}}{\partial \boldsymbol{n}_{X}} .
$$

In a similar manner the variation of the electrodynamic field Lagrangian $\Lambda_{\mathrm{EM}}$ defines the so-called "electric displacement field" $\boldsymbol{D}$ and the "magnetic field strength" $\boldsymbol{H}$ as conjugate variables to the electromagnetic fields, namely

$$
d \Lambda_{\mathrm{EM}}=\frac{1}{4 \pi} \boldsymbol{D} \cdot d \boldsymbol{E}-\frac{1}{4 \pi} \boldsymbol{H} \cdot d \boldsymbol{B}
$$

and using the explicit form (10) we find

$$
\boldsymbol{D}=4 \pi \frac{\partial \Lambda_{\mathrm{EM}}}{\partial \boldsymbol{E}}=\boldsymbol{E}, \quad \boldsymbol{H}=-4 \pi \frac{\partial \Lambda_{\mathrm{EM}}}{\partial \boldsymbol{B}}=\boldsymbol{B} .
$$

Although we can trivially identify $\boldsymbol{D}=\boldsymbol{E}$ and $\boldsymbol{H}=\boldsymbol{B}$ in the present non-polarizable case, in the following we nevertheless keep the formal and conceptual separation between the "kinematical" $\boldsymbol{E}$ and $\boldsymbol{B}$ and the "dynamical" (i.e. derived from $\Lambda$ ) quantities $\boldsymbol{D}$ and $\boldsymbol{H}$.

Due to the presence of the minimal coupling terms in (8), the variation of the total Lagrangian $\Lambda$ generalizes the dynamical momenta $p_{0}^{X}$ and $\boldsymbol{p}^{X}$ to the (gauge-dependent) canonical momenta $\pi_{0}^{X}$ and $\boldsymbol{\pi}^{X}$, namely

$$
d \Lambda=\sum\left(\pi_{0}^{X} d n_{X}+\boldsymbol{\pi}^{X} \cdot d \boldsymbol{n}_{X}\right)-\rho d \Phi+\sigma d A_{0}+\frac{1}{c} \boldsymbol{j} \cdot d \boldsymbol{A}+d \Lambda_{\mathrm{EM}}+d \Lambda_{\mathrm{grav}}
$$

from which we obtain the relations

$$
\begin{aligned}
& \pi_{0}^{X}=p_{0}^{X}+q^{X} A_{0}-m^{X} \Phi \\
& \boldsymbol{\pi}^{X}=\boldsymbol{p}^{X}+\frac{1}{c} q^{X} \boldsymbol{A},
\end{aligned}
$$

expressing the canonical momenta in terms of the dynamical momenta and the gauge fields.

\section{The equations of motion}

We see from definition (5) that the first two Maxwell equations are satisfied identically, i.e.

$$
\nabla \cdot \boldsymbol{B}=0, \quad \text { and } \quad \nabla \times \boldsymbol{E}+\frac{1}{c} \partial_{t} \boldsymbol{B}=0 .
$$

Using (12) and (14) it is not difficult to show that requiring invariance of the action (7) under free variations $\delta A_{0}$ and $\delta \boldsymbol{A}$ of the gauge fields results in the two remaining Maxwell equations

$$
\nabla \cdot \boldsymbol{D}=4 \pi \sigma, \quad \text { and } \quad \nabla \times \boldsymbol{H}-\frac{1}{c} \partial_{t} \boldsymbol{D}=\frac{4 \pi}{c} \boldsymbol{j} .
$$

Similarly, requiring invariance of the action (7) with respect to free infinitesimal variations $\delta \Phi$ straightforwardly results in the Poisson equation for the gravitational field,

$$
\nabla^{2} \Phi=4 \pi G \rho .
$$


The derivation of the equations of motion for the fluids is formally completely equivalent to the uncoupled case discussed in Paper I. The only difference is that the dynamical momenta $p_{0}^{X}$ and $\boldsymbol{p}^{X}$ are now replaced by the corresponding canonical momenta $\pi_{0}^{X}$ and $\pi^{X}$.

One has to consider infinitesimal spatial displacements $\boldsymbol{\xi}_{X}$ and time-shifts $\tau_{X}$ of the flowlines, which should be considered the "true" fundamental quantities describing hydrodynamics (corresponding to the Lagrangian framework), while free variations for densities and velocities (characteristic for the Eulerian framework) fail to produce the correct equations, except when adding "ad-hoc" constraints to the Lagrangian. The Eulerian variables, density $n_{X}$ and velocity $\boldsymbol{v}_{X}$ can be expressed in terms of the underlying flowlines (and initial conditions) alone, and one should therefore also consider the hydrodynamic Lagrangian $\Lambda_{\mathrm{H}}$ in (8) as a function of these variables. These subtleties relating to the hydrodynamic variational principle are discussed in greater detail in Paper I.

The resulting induced variations of the densities, $\delta n_{X}$, and currents, $\delta \boldsymbol{n}_{X}$ have been derived in Paper I as

$$
\begin{aligned}
\delta n_{X} & =-\nabla \cdot\left[n_{X} \boldsymbol{\xi}_{X}\right]+\left[\boldsymbol{n}_{X} \cdot \nabla \tau_{X}-\tau_{X} \partial_{t} n_{X}\right], \\
\delta \boldsymbol{n}_{X} & =n_{X} \partial_{t} \boldsymbol{\xi}_{X}+\left(\boldsymbol{n}_{X} \cdot \nabla\right) \boldsymbol{\xi}_{X}-\left(\boldsymbol{\xi}_{X} \cdot \nabla\right) \boldsymbol{n}_{X}-\boldsymbol{n}_{X}\left(\nabla \cdot \boldsymbol{\xi}_{X}\right)-\partial_{t}\left(\boldsymbol{n}_{X} \tau_{X}\right) .
\end{aligned}
$$

Substituting these expressions into the variation of the total Lagrangian (14) and integrating by parts, we arrive at the following form of the induced action variation

$$
\delta \mathcal{I}=\int \sum\left(g^{X} \tau_{X}-\boldsymbol{f}^{X} \cdot \boldsymbol{\xi}_{X}\right) d V d t,
$$

where the canonical force densities $\boldsymbol{f}^{X}$ (acting on the constituent) and the energy transfer rates $g^{X}$ (into the constituent) are found explicitly as

$$
\begin{aligned}
\boldsymbol{f}^{X} & =n_{X}\left(\partial_{t} \boldsymbol{\pi}^{X}-\nabla \pi_{0}^{X}\right)-\boldsymbol{n}_{X} \times\left(\nabla \times \boldsymbol{\pi}^{X}\right)+\boldsymbol{\pi}^{X} \Gamma_{X}, \\
g^{X} & =\boldsymbol{v}_{X} \cdot\left(\boldsymbol{f}^{X}-\boldsymbol{\pi}^{X} \Gamma_{X}\right)-\pi_{0}^{X} \Gamma_{X},
\end{aligned}
$$

where $\Gamma_{X}$ is the particle creation rate for the constituent $X$, i.e.

$$
\Gamma_{X} \equiv \partial_{t} n_{X}+\nabla \cdot \boldsymbol{n}_{X}
$$

The canonical force density $\boldsymbol{f}^{X}$ is the total (canonical) momentum change rate of the constituent $X$, and the last term in (23) represents a contribution that is purely due to the change of the particle number. By inserting the explicit expressions (1516) of the canonical momenta into (23) and (24), we can separate out the purely hydrodynamic contribution $\boldsymbol{f}_{\mathrm{H}}^{X}$, which expresses fluid inertia and pressure, and which has the same form as in the uncharged case (Paper I), namely

$$
\boldsymbol{f}_{\mathrm{H}}^{X} \equiv n_{X}\left(\partial_{t} \boldsymbol{p}^{X}-\nabla p_{0}^{X}\right)-\boldsymbol{n}_{X} \times\left(\nabla \times \boldsymbol{p}^{X}\right) .
$$

Introducing the gravitational and electromagnetic forces $\boldsymbol{f}_{\mathrm{grav}}^{X}$ and $\boldsymbol{f}_{\mathrm{EM}}^{X}$, defined by their usual expressions

$$
\begin{aligned}
\boldsymbol{f}_{\mathrm{grav}}^{X} & =-n_{X} m^{X} \nabla \Phi, \\
\boldsymbol{f}_{\mathrm{EM}}^{X} & =n_{X} q^{X}\left(\boldsymbol{E}+\frac{1}{c} \boldsymbol{v}_{X} \times \boldsymbol{B}\right),
\end{aligned}
$$

we can now rewrite the canonical force densities (23) in the form

$$
\boldsymbol{f}^{X}=\boldsymbol{f}_{\mathrm{H}}^{X}-\boldsymbol{f}_{\mathrm{grav}}^{X}-\boldsymbol{f}_{\mathrm{EM}}^{X}+\Gamma_{X} \boldsymbol{\pi}^{X}
$$

The actual physical equations of motion are obtained by prescribing the canonical force densities $\boldsymbol{f}^{X}$ acting on the fluids. If we only require invariance of the action for a common displacement and time shift $\boldsymbol{\xi}_{X}=\boldsymbol{\xi}_{\text {and }} \tau_{X}=\tau$, we obtain the minimal equations of motion for the total system, namely

$$
\sum \boldsymbol{f}^{X}=\boldsymbol{f}_{\text {ext }}, \text { and } \sum g^{X}=g_{\text {ext }}
$$

where $\boldsymbol{f}_{\text {ext }}$ and $g_{\text {ext }}$ are interpretable as the "external" force density and energy transfer rate acting on the system. This generalizes the more common action principle of isolated systems, in which the external influences $\boldsymbol{f}_{\text {ext }}$ and $g_{\text {ext }}$ vanish and therefore the equations of motion would be obtained by requiring the action to be invariant under small variations. "External" here is meant in the sense of not being included in the total Lagrangian, which could also include, for example, viscous forces. 


\section{The hydrodynamic Lagrangian $\Lambda_{\mathrm{H}}$}

As shown in Paper I, the hydrodynamic Lagrangian density $\Lambda_{\mathrm{H}}$ for the class of "perfect" (i.e. with an isotropic energy function $\mathcal{E}$ ) multi-fluid systems is given by

$$
\Lambda_{\mathrm{H}}\left(n_{X}, \boldsymbol{n}_{X}\right)=\sum m^{X} \frac{\boldsymbol{n}_{X}^{2}}{2 n_{X}}-\mathcal{E}\left(n_{X}, \Delta_{X Y}^{2}\right),
$$

where $\boldsymbol{\Delta}_{X Y}$ denotes the relative velocity between fluids $X$ and $Y$ as defined in (11). The total differential of the thermodynamic potential $\mathcal{E}\left(n_{X}, \Delta_{X Y}^{2}\right)$ determines the first law of thermodynamics for the matter-system, namely

$$
d \mathcal{E}=\sum \mu^{X} d n_{X}+\frac{1}{2} \sum_{X, Y} \alpha^{X Y} d \Delta_{X Y}^{2}
$$

which defines the chemical potentials $\mu^{X}$ and the symmetric entrainment matrix $\alpha^{X Y}$. The dynamical momenta $p_{0}^{X}$ and $\boldsymbol{p}^{X}$ defined in (11) are therefore found as

$$
\begin{aligned}
\boldsymbol{p}^{X} & =m^{X} \boldsymbol{v}_{X}-\sum_{Y} \frac{2 \alpha^{X Y}}{n_{X}} \boldsymbol{\Delta}_{X Y}, \\
-p_{0}^{X} & =\mu^{X}-m^{X} \frac{v_{X}^{2}}{2}+\boldsymbol{v}_{X} \cdot \boldsymbol{p}^{X} .
\end{aligned}
$$

\section{GAUSS-TYPE CONSERVATION LAWS}

\section{A. Conservation of charge and mass}

We see from (26) - 28 that the force contributions $\boldsymbol{f}_{\mathrm{H}}^{X}, \boldsymbol{f}_{\mathrm{grav}}^{X}$ and $\boldsymbol{f}_{\mathrm{EM}}^{X}$ are invariant with respect to gauge transformations of the gravitational and electromagnetic fields. However, the last term in the expressions (23) and (24) (accounting for momentum and energy change due to particle number changes) is generally gauge dependent. While this is not prohibited for individual constituent forces, the total equations of motion (30) have to be gauge invariant, and by using (1516) we can therefore deduce the constraints

$$
\begin{aligned}
\sum q^{X} \Gamma_{X} & =\partial_{t} \sigma+\nabla \cdot \boldsymbol{j}=0, \\
\sum m^{X} \Gamma_{X} & =\partial_{t} \rho+\nabla \cdot \boldsymbol{\rho}=0,
\end{aligned}
$$

where the total densities and currents have been defined in (2) and (3). Gauge invariance therefore implies conservation of the "charge" associated with the gauge field. As a further consequence we find the following useful relations,

$$
\sum \Gamma_{X} \boldsymbol{\pi}^{X}=\sum \Gamma_{X} \boldsymbol{p}^{X}, \text { and } \sum \Gamma_{X} \pi_{0}^{X}=\sum \Gamma_{X} p_{0}^{X}
$$

We note that the conservation of mass was already derived in Paper I as a consequence of Galilean invariance, which can also be considered a gauge freedom.

\section{B. Momentum conservation}

As shown in Paper I, the purely hydrodynamic force densities $\boldsymbol{f}_{\mathrm{H}}^{X}$ satisfy the relation

$$
\sum\left(f_{\mathrm{H}}^{X i}+\Gamma_{X} p^{X i}\right)=\partial_{t} J_{\mathrm{H}}^{i}+\nabla_{j} T_{\mathrm{H}}^{i j},
$$

where the hydrodynamic momentum density $\boldsymbol{J}_{\mathrm{H}}$ and stress tensor $T_{\mathrm{H}}^{i j}$ are given by

$$
\boldsymbol{J}_{\mathrm{H}} \equiv \sum n_{X} \boldsymbol{p}^{X}, \quad \text { and } \quad T_{\mathrm{H}}^{i j} \equiv \sum n_{X}^{i} p^{X j}+\Psi g^{i j} .
$$

The "generalized pressure" $\Psi$ is defined via the Legendre transformation of $\Lambda_{\mathrm{H}}$, namely

$$
\Psi \equiv \Lambda_{\mathrm{H}}-\sum\left(n_{X} p_{0}^{X}+\boldsymbol{n}_{X} \cdot \boldsymbol{p}^{X}\right)
$$


and $g_{i j}$ are the components of the metric tensor, which in Cartesian coordinates is simply $g_{i j}=\delta_{i j}$. Using these relations together with (29) and (37), we can write the total force balance equation (30) in the form

$$
f_{\mathrm{ext}}^{i}=\sum f^{X i}=\partial_{t} J_{\mathrm{H}}^{i}+\nabla_{j} T_{\mathrm{H}}^{i j}-f_{\mathrm{grav}}^{i}-f_{\mathrm{EM}}^{i}
$$

where $\boldsymbol{f}_{\text {grav }} \equiv \sum \boldsymbol{f}_{\mathrm{grav}}^{X}$ and $\boldsymbol{f}_{\mathrm{EM}} \equiv \sum \boldsymbol{f}_{\mathrm{EM}}^{X}$ are the total gravitational and electromagnetic force densities. Using (27) and (28) we find explicitly

$$
\begin{aligned}
\boldsymbol{f}_{\text {grav }} & =-\rho \nabla \Phi \\
\boldsymbol{f}_{\mathrm{EM}} & =\sigma \boldsymbol{E}+\frac{1}{c} \boldsymbol{j} \times \boldsymbol{B} .
\end{aligned}
$$

One can easily verify that the gravitational force term can be written as the divergence of a tensor, namely

$$
-f_{\text {grav }}^{i}=\nabla_{j} T_{\text {grav }}^{i j}, \quad \text { with } \quad T_{\text {grav }}^{i j}=\frac{1}{4 \pi G}\left(\nabla^{i} \Phi \nabla^{j} \Phi-\frac{1}{2}(\nabla \Phi)^{2} g^{i j}\right) .
$$

Using the Maxwell equations (17), (18) and the total differential (12) of $\Lambda_{\mathrm{EM}}$, we can show that the total electromagnetic force density (43) can be similarly rewritten as

$$
-f_{\mathrm{EM}}^{i}=\partial_{t} J_{\mathrm{EM}}^{i}+\nabla_{j} T_{\mathrm{EM}}^{i j}
$$

in terms of the momentum density $\boldsymbol{J}_{\mathrm{EM}}$ of the electromagnetic field,

$$
\boldsymbol{J}_{\mathrm{EM}} \equiv \frac{1}{4 \pi c} \boldsymbol{D} \times \boldsymbol{B},
$$

and the Maxwell stress tensor $T_{\mathrm{EM}}^{i j}$, which is found as

$$
T_{\mathrm{EM}}^{i j}=-\frac{1}{4 \pi}\left(E^{i} D^{j}+H^{i} B^{j}\right)+\left(\Lambda_{\mathrm{EM}}+\frac{1}{4 \pi} \boldsymbol{H} \cdot \boldsymbol{B}\right) g^{i j} .
$$

Putting all the pieces together, we obtain the following form for the total momentum conservation (41):

$$
\partial_{t}\left(J_{\mathrm{H}}^{i}+J_{\mathrm{EM}}^{i}\right)+\nabla_{j} T^{i j}=f_{\mathrm{ext}}^{i},
$$

where the total stress tensor is given by

$$
T^{i j} \equiv T_{\mathrm{H}}^{i j}+T_{\mathrm{grav}}^{i j}+T_{\mathrm{EM}}^{i j}
$$

An important property of the total stress tensor $T^{i j}$ is that it is symmetric. The symmetry of the gravitational part (44) is obvious, while the symmetry of the hydrodynamic stress tensor (39) has been shown in Paper I. It remains to prove the symmetry of the electromagnetic stress tensor (47). In the present non-polarizable case, this follows trivially from the identities (13). It is interesting to note, however, that this can also be derived more generally as a Noether identity of the variational principle, assuming only the separable form (8) of the Lagrangian.

In order to show this, we extend the variational principle slightly by admitting also metric variations $\delta g_{i j}$, so that (12) now reads as

$$
\delta \Lambda_{\mathrm{EM}}=\frac{1}{4 \pi} \boldsymbol{D} \cdot \delta \boldsymbol{E}-\frac{1}{4 \pi} \boldsymbol{H} \cdot \delta \boldsymbol{B}+\frac{\partial \Lambda_{\mathrm{EM}}}{\partial g_{i j}} \delta g_{i j}
$$

Consider an active time-independent infinitesimal displacement $\boldsymbol{\xi}$ of the whole system including the metric, which induces the following Lagrangian changes:

$$
\Delta E_{i}=E^{l} \nabla_{i} \xi_{l}, \quad \Delta B_{i}=B^{l} \nabla_{i} \xi_{l}, \quad \Delta g_{i j}=-2 \nabla_{(i} \xi_{j)} .
$$

Using these transformations together with (50), we obtain the induced Lagrangian change of $\Lambda_{\mathrm{EM}}$ as

$$
\Delta \Lambda_{\mathrm{EM}}=\frac{1}{4 \pi}\left[D^{i} E^{j}-H^{i} B^{j}-8 \pi \frac{\partial \Lambda_{\mathrm{EM}}}{\partial g_{i j}}\right] \nabla_{i} \xi_{j} .
$$


This active transformation is equivalent to a coordinate transformation $-\boldsymbol{\xi}$, and therefore the requirement of $\Lambda_{\mathrm{EM}}$ being a scalar is $\Delta \Lambda_{\mathrm{EM}}=0$, which leads to the associated Noether identity

$$
2 \frac{\partial \Lambda_{\mathrm{EM}}}{\partial g_{i j}}=\frac{1}{4 \pi}\left[D^{i} E^{j}-H^{i} B^{j}\right] .
$$

Using the manifest symmetry of the left-hand side, we obtain

$$
E^{i} D^{j}+H^{i} B^{j}=E^{j} D^{i}+H^{j} B^{i}
$$

which concludes the proof of the symmetry of $T_{\mathrm{EM}}^{i j}$. Note that in general $T_{\mathrm{EM}}^{i j}$ need not be symmetric, only the sum of all contributions to $T^{i j}$ is subject to this constraint. The symmetry of $T_{\mathrm{EM}}^{i j}$ is a special consequence of the assumption of a "separable" interaction of the form (8).

\section{Energy conservation}

We have seen in Paper I that we can write

$$
\sum\left(\boldsymbol{v}_{X} \cdot \boldsymbol{f}_{\mathrm{H}}^{X}-\Gamma_{X} p_{0}^{X}\right)=\partial_{t} E_{\mathrm{H}}+\nabla \cdot \boldsymbol{Q}_{\mathrm{H}}
$$

in terms of the hydrodynamic energy density $E_{\mathrm{H}}$ and energy flux $\boldsymbol{Q}_{\mathrm{H}}$, which are given by

$$
E_{\mathrm{H}}=\sum \boldsymbol{n}_{X} \cdot \boldsymbol{p}^{X}-\Lambda_{\mathrm{H}}, \quad \text { and } \quad \boldsymbol{Q}_{\mathrm{H}}=\sum\left(-p_{0}^{X}\right) \boldsymbol{n}_{X},
$$

while for the gravitational and electromagnetic work contributions $g_{\text {grav }} \equiv \sum \boldsymbol{v}_{X} \cdot \boldsymbol{f}_{\mathrm{grav}}^{X}$ and $g_{\mathrm{EM}} \equiv \sum \boldsymbol{v}_{X} \cdot \boldsymbol{f}_{\mathrm{EM}}^{X}$, we find using (27) and (28):

$$
\begin{aligned}
g_{\mathrm{grav}} & =-\boldsymbol{\rho} \cdot \nabla \Phi, \\
g_{\mathrm{EM}} & =\boldsymbol{j} \cdot \boldsymbol{E} .
\end{aligned}
$$

Using these expressions, the energy equation (30) can be written as

$$
g_{\mathrm{ext}}=\partial_{t} E_{\mathrm{H}}+\nabla \cdot \boldsymbol{Q}_{\mathrm{H}}-g_{\mathrm{grav}}-g_{\mathrm{EM}} .
$$

Using Maxwell's equations (17) and (18), one can write the electric work $g_{\mathrm{EM}}$ in the form of a conservation law, namely

$$
-g_{\mathrm{EM}}=\partial_{t} E_{\mathrm{EM}}+\nabla \cdot \boldsymbol{S}
$$

where the electromagnetic field energy density $E_{\mathrm{EM}}$ is given by

$$
E_{\mathrm{EM}}=\frac{1}{4 \pi} \boldsymbol{E} \cdot \boldsymbol{D}-\Lambda_{\mathrm{EM}}=\frac{1}{8 \pi}\left(\boldsymbol{E}^{2}+\boldsymbol{B}^{2}\right),
$$

and the second equality was obtained using the explicit Lagrangian (10). The energy flux $\boldsymbol{S}$ is given by the Poynting vector

$$
\boldsymbol{S}=\frac{c}{4 \pi} \boldsymbol{E} \times \boldsymbol{H} .
$$

In the present non-polarizable case, i.e. $\boldsymbol{D}=\boldsymbol{E}$ and $\boldsymbol{B}=\boldsymbol{H}$, we recover the well-known relation between the energy flux and momentum of the electromagnetic field, namely $\boldsymbol{J}_{\mathrm{EM}}=\boldsymbol{S} / c^{2}$. Summarizing, we can cast (59) in the form of a conservation of total energy, namely

$$
\partial_{t}\left(E_{\mathrm{H}}+E_{\mathrm{EM}}\right)+\nabla \cdot\left(\boldsymbol{Q}_{\mathrm{H}}+\boldsymbol{S}\right)=g_{\mathrm{ext}}-\boldsymbol{\rho} \cdot \nabla \Phi
$$

We note that formally one can also write the gravitational work in the form of a conservation law, but the expression for energy density and flux are neither unique nor gauge invariant, and one can also not eliminate the mass current $\boldsymbol{\rho}$ from these expressions due to the lack of a dynamic law for the gravitational field in the Newtonian framework. 


\section{CONSERVATION ALONG FLOWLINES}

In this section we show how the conservation of vorticity and helicity, derived for uncharged fluids in Paper I, can be generalized quite naturally to the case of fluids coupled to the electromagnetic and gravitational field. We note that the technical steps involved in this discussion are largely analogous to the treatment in Paper I, and we therefore skip most intermediate steps.

\section{A. Generalized Kelvin-Helmholtz vorticity conservation}

We define the hydrodynamic vorticity 2 -form $\underline{w}$ as the exterior derivative of the dynamical momentum 1 -form $\underline{p}$, namely $\underline{w} \equiv d p$, and the more common dual vorticity vector $\boldsymbol{W}$, which is $\boldsymbol{W}=\nabla \times \boldsymbol{p}$.

In the presence of electromagnetic fields, the more fundamental quantity is the canonical vorticity 2 -form $\underline{\varpi}$, which is defined in the same way but with respect to the canonical momentum $\underline{\pi}$, namely

$$
\underline{\varpi} \equiv d \underline{\pi},
$$

and the dual canonical vorticity vector $\mathcal{W}$ is therefore given by

$$
\mathcal{W}=\nabla \times \pi
$$

With (16) we see that the relation between canonical and hydrodynamic vorticity is simply

$$
\underline{\varpi}=\underline{w}+\frac{q}{c} d \underline{A}, \quad \text { and } \quad \mathcal{W}=\boldsymbol{W}+\frac{q}{c} \boldsymbol{B} .
$$

We note that by the Poincaré property (namely $d d=0$ ), the exterior derivatives of the vorticity 2-forms vanish identically, i.e. $d \underline{\varpi}=0$, which equivalently expresses the fact that the vorticity vectors are divergence-free, i.e. $\nabla \cdot \mathcal{W}=0$

We can write the expression (23) for the canonical force $\boldsymbol{f}$ acting on one constituent in the language of forms as

$$
\left.\partial_{t} \underline{\pi}+\boldsymbol{v}\right\rfloor d \underline{\pi}-d \pi_{0}=\frac{1}{n}(\underline{f}-\Gamma \underline{\pi})
$$

where 」 indicates summation over adjacent vector- and form-indices.

In the following it will be convenient to separate the "proper force" per particle acting on the right-hand side of (67) into its non-conservative part $\underline{\mathfrak{F}}$ and a conservative contribution $d \phi$, namely

$$
\frac{1}{n}(\underline{f}-\Gamma \underline{\pi})=d \phi+\underline{\mathfrak{F}}
$$

Applying the Cartan formula for the Lie derivative of a $p$-form to the 1-form $\underline{\pi}$, namely $£ \boldsymbol{v} \underline{\pi}=\boldsymbol{v}\rfloor d \underline{\pi}+d(\boldsymbol{v}\rfloor \underline{\pi})$, allows us now to rewrite the force equation (67) more conveniently as

$$
\left(\partial_{t}+£_{\boldsymbol{v}}\right) \underline{\pi}=d Q+\underline{\mathfrak{F}},
$$

where the scalar $Q$ is given by $\left.Q=\pi_{0}+\boldsymbol{v}\right\rfloor \underline{\pi}+\phi$. Lie derivatives and partial time derivatives commute with exterior derivatives, so we can apply an exterior derivative to (69) and with (64) we obtain the Helmholtz equation of transport of canonical vorticity, namely

$$
\left(\partial_{t}+£_{\boldsymbol{v}}\right) \underline{\varpi}=d \underline{F}
$$

which shows that the canonical vorticity is conserved under transport by the fluid, if the proper force per particle acting on the fluid is purely conservative, i.e. if $\underline{\mathfrak{F}}=0$. In its more common dual form, this equation can be written as

$$
\partial_{t} \mathcal{W}-\nabla \times(\boldsymbol{v} \times \mathcal{W})=\nabla \times \mathfrak{F}
$$

Substituting the explicit relation (66) between the dynamical and the canonical vorticity, and using Maxwell's equation, this can be re-expressed as

$$
\partial_{t} \boldsymbol{W}-\nabla \times(\boldsymbol{v} \times \boldsymbol{W})=\nabla \times\left[\mathfrak{F}+q\left(\boldsymbol{E}+\frac{1}{c} \boldsymbol{v} \times \boldsymbol{B}\right)\right]
$$


which shows that in the case of a charged constituent, the dynamical vorticity $\boldsymbol{W}$ is generally not conserved even in the absence of a non-conservative external force $\mathfrak{F}$, due to the presence of the Lorentz force acting on the fluid. However, the canonical vorticity is conserved in this case and therefore generalizes the vorticity conservation of uncharged fluids.

For the canonical circulation $\mathcal{C}$ of a closed circuit $\partial \Sigma$, which is the boundary of a 2 -surface $\Sigma$, we have

$$
\mathcal{C} \equiv \oint_{\partial \Sigma} \underline{\pi}=\int_{\Sigma} \underline{\varpi}=\int_{\Sigma} \mathcal{W} \cdot d \boldsymbol{S}
$$

where $d \boldsymbol{S}$ is the surface normal element. We see from (66) that the canonical circulation $\mathcal{C}$ can also be expressed as the sum of the hydrodynamic vorticity flux (i.e. dynamical circulation) and the magnetic flux through the surface $\Sigma$, namely

$$
\mathcal{C}=\int_{\Sigma} \boldsymbol{W} \cdot d \boldsymbol{S}+\frac{q}{c} \int_{\Sigma} \boldsymbol{B} \cdot d \boldsymbol{S} .
$$

For the comoving time derivative of the circulation $\mathcal{C}$ we find using (69)

$$
\frac{d \mathcal{C}}{d t}=\oint\left(\partial_{t}+£_{\boldsymbol{v}}\right) \underline{\pi}=\oint_{\partial \Sigma} \underline{\mathfrak{F}}
$$

which is Kelvin's theorem for the conservation of canonical circulation. We note that strict conservation only applies if the non-conservative force per particle $\underline{\mathfrak{F}}$ vanishes, as we have already seen earlier.

\section{B. Vorticity and superconductors}

As discussed in more detail in Paper I, the hydrodynamics of superfluids is generally characterized by two fundamental properties: the absence of dissipative mechanisms like friction or viscosity, and the constraint of irrotational flow. While in the case of uncharged superfluids this simply meant the vanishing of the dynamical vorticity $\boldsymbol{W}$, it is now the canonical vorticity $\mathcal{W}$ that is constrained to vanish identically in the case of charged superfluids, more commonly referred to as "superconductors". The absence of microscopic dissipative mechanisms implies that there is no non-conservative force acting on the bulk ${ }^{2}$ of the superfluid, i.e.

$$
\mathfrak{F}^{\mathrm{S}}=0,
$$

which quite generally characterizes perfect conductors of any sort. As a consequence we see that the canonical vorticity (and equivalently circulation) of a perfect conductor is strictly conserved, as seen in the previous section. The further constraint of irrotational flow, which distinguishes a superconductor from a mere perfect conductor, reads as

$$
\underline{\varpi}^{\mathrm{S}}=\underline{w}^{\mathrm{S}}+\frac{1}{c} q^{\mathrm{S}} d \underline{A}=0, \quad \text { and } \quad \mathcal{W}^{\mathrm{S}}=\boldsymbol{W}^{\mathrm{S}}+\frac{1}{c} q^{\mathrm{S}} \boldsymbol{B}=0 .
$$

We see from (70) or (71) that if this irrotationality constraint is satisfied at some instant $t$, then it will automatically remain true for all subsequent times due to the absence of dissipation (76). We can therefore write the superfluid momentum $\underline{\pi}^{\mathrm{S}}$ (locally) as the gradient of a phase $\varphi$, i.e.

$$
\boldsymbol{\pi}^{\mathrm{S}}=\boldsymbol{p}^{\mathrm{S}}+\frac{q^{\mathrm{S}}}{c} \boldsymbol{A}=\hbar \nabla \varphi,
$$

which leads to the well-known London equation for superconductors, as further discussed in Sect. $\mathrm{VC}$ The canonical circulation (73) can therefore be non-zero if $\partial \Sigma$ encloses a topological defect in the phase $\varphi$, i.e. a region where $\varphi$ (and therefore $\pi^{\mathrm{S}}$ ) is not defined, as for example in the case of flow inside a torus, or around a vortex. While in the case of a perfect irrotational fluid the resulting circulation could have any value, the superfluid phase $\varphi$ is restricted to change by a multiple of $2 \pi$ when following a closed loop inside the superfluid around the defect. The resulting canonical circulation is therefore quantized as

$$
\mathcal{C}=2 N \pi \hbar, \quad \text { with } \quad N \in \mathbb{Z},
$$

which gives rise to the well-known quantized vortex structure and flux quantization of superconductors.

\footnotetext{
2 As mentioned in Paper I, this condition can be violated in the core of vortices, leading to "mutual friction".
} 


\section{Generalized helicity conservation}

We now turn to the generalization of the dynamical helicity conservation derived in Paper I. We define the canonical helicity 3 -form $\underline{H}$ as the exterior product of the momentum 1-form $\underline{\pi}$ with the vorticity 2 -form $\underline{\varpi}$, i.e.

$$
\underline{H} \equiv \underline{\pi} \wedge \underline{\varpi}
$$

and we define the dual canonical helicity density $h$ as

$$
\underline{H}=h \underline{\epsilon},
$$

where $\underline{\epsilon}$ is the volume form with components $\epsilon_{i j k}$. The helicity scalar can be seen to have the following explicit expressions

$$
h=\underline{\pi}\rfloor \mathcal{W}=\pi \cdot(\nabla \times \pi) .
$$

Using (69) and (70), the comoving time-derivative of $\underline{H}$ can be found as

$$
\left(\partial_{t}+£_{\boldsymbol{v}}\right) \underline{H}=d(Q \underline{\varpi})+[d(\underline{\pi} \wedge \underline{\mathfrak{F}})+2 d \underline{\mathfrak{F}} \wedge \underline{\pi}] .
$$

If we further introduce the total canonical helicity $\mathcal{H}$ of a volume $V$ as

$$
\mathcal{H} \equiv \int_{V} \underline{H}=\int_{V} h d V,
$$

then we find in the absence of non-conservative forces, i.e. $\underline{\mathfrak{F}}=0$, that the comoving time derivative of $\mathcal{H}$ satisfies

$$
\frac{d \mathcal{H}}{d t}=\oint_{\partial V} Q \mathcal{W} \cdot d \boldsymbol{S}
$$

Therefore the canonical helicity $\mathcal{H}$ of a volume $V$ is conserved under transport by the fluid only if, in addition to $\underline{\mathfrak{F}}=0$, the canonical vorticity $\mathcal{W}$ vanishes on the surface $\partial V$ surrounding this volume. We note that in general the conserved helicity $\mathcal{H}$ contains contributions from the purely hydrodynamic "Moffat" helicity $\boldsymbol{p} \cdot \boldsymbol{W}$ and the magnetic helicity $\boldsymbol{A} \cdot \boldsymbol{B}$ together with "mixed" terms, namely using (82) we can express

$$
h=\boldsymbol{p} \cdot \boldsymbol{W}+\frac{q^{2}}{c^{2}} \boldsymbol{A} \cdot \boldsymbol{B}+\frac{q}{c}[\boldsymbol{p} \cdot \boldsymbol{B}+\boldsymbol{A} \cdot \boldsymbol{W}] .
$$

\section{APPLiCATiONS}

\section{A. General description of electric conductors}

As a simple application of the foregoing formalism, we consider an electric conductor describable as a two-constituent system. One constituent consists of the positively charged ions, described by their number density $n$, velocity $\boldsymbol{v}$, mass per ion $m$ and charge per ion $q=Z e$. The second constituent is a gas of electrons of density $n_{\mathrm{e}}$, velocity $\boldsymbol{v}_{\mathrm{e}}$, mass $m_{\mathrm{e}}$ and charge $q^{\mathrm{e}}=-e$. The total charge density and current (3) are therefore expressible as

$$
\sigma=e\left(Z n-n_{\mathrm{e}}\right), \quad \text { and } \quad \boldsymbol{j}=e\left(Z n \boldsymbol{v}-n_{\mathrm{e}} \boldsymbol{v}_{\mathrm{e}}\right)
$$

and the relative velocity between the two fluids is

$$
\Delta \equiv \boldsymbol{v}-\boldsymbol{v}_{\mathrm{e}}
$$

Charge transfer between the two fluids is possible in principle, e.g. we could allow for processes of ionization and recombination, where electrons are transferred from the ion-fluid to the fluid of free electrons. But for simplicity we will assume the number of free electrons to be conserved, so we have

$$
\Gamma=\partial_{t} n+\nabla \cdot(n \boldsymbol{v})=0, \quad \text { and } \quad \Gamma_{e}=\partial_{t} n_{\mathrm{e}}+\nabla \cdot\left(n_{\mathrm{e}} \boldsymbol{v}_{\mathrm{e}}\right)=0 .
$$

The total differential of the energy function $\mathcal{E}\left(n, n_{\mathrm{e}}, \boldsymbol{\Delta}^{2}\right)$ is

$$
d \mathcal{E}=\mu d n+\mu^{\mathrm{e}} d n_{\mathrm{e}}+\alpha d \Delta^{2}
$$


Using (33) and (34), the conjugate momenta of electrons and ions are therefore found as

$$
\begin{aligned}
& \boldsymbol{p}=m \boldsymbol{v}-\frac{2 \alpha}{n} \boldsymbol{\Delta}, \quad-p_{0}=\mu-\frac{1}{2} m v^{2}+\boldsymbol{v} \cdot \boldsymbol{p}, \\
& \boldsymbol{p}^{\mathrm{e}}=m_{\mathrm{e}} \boldsymbol{v}_{\mathrm{e}}+\frac{2 \alpha}{n_{\mathrm{e}}} \boldsymbol{\Delta}, \quad-p_{0}^{\mathrm{e}}=\mu^{\mathrm{e}}-\frac{1}{2} m_{\mathrm{e}} v_{\mathrm{e}}^{2}+\boldsymbol{v}_{\mathrm{e}} \cdot \boldsymbol{p}^{\mathrm{e}} .
\end{aligned}
$$

We neglect the gravitational field, so $\boldsymbol{f}_{\text {grav }}=0$, and the canonical force densities acting on the electron- and ion-fluid are obtained from (28) and (29) as

$$
\boldsymbol{f}=\boldsymbol{f}_{\mathrm{H}}-n Z e\left(\boldsymbol{E}+\frac{\boldsymbol{v}}{c} \times \boldsymbol{B}\right), \quad \text { and } \quad \boldsymbol{f}^{\mathrm{e}}=\boldsymbol{f}_{\mathrm{H}}^{\mathrm{e}}+e n_{\mathrm{e}}\left(\boldsymbol{E}+\frac{\boldsymbol{v}_{\mathrm{e}}}{c} \times \boldsymbol{B}\right),
$$

where the hydrodynamic force densities are obtained from (26), by substituting the dynamical momenta (91), which yields

$$
\begin{aligned}
& \boldsymbol{f}_{\mathrm{H}}=n m\left(\partial_{t}+\boldsymbol{v} \cdot \nabla\right)\left[\boldsymbol{v}-\frac{2 \alpha}{n m} \boldsymbol{\Delta}\right]+n \nabla \mu-2 \alpha \Delta_{j} \nabla v^{j}, \\
& \boldsymbol{f}_{\mathrm{H}}^{\mathrm{e}}=n_{\mathrm{e}} m_{\mathrm{e}}\left(\partial_{t}+\boldsymbol{v}_{\mathrm{e}} \cdot \nabla\right)\left[\boldsymbol{v}_{\mathrm{e}}+\frac{2 \alpha}{n_{\mathrm{e}} m_{\mathrm{e}}} \boldsymbol{\Delta}\right]+n_{\mathrm{e}} \nabla \mu^{\mathrm{e}}+2 \alpha \Delta_{j} \nabla v_{\mathrm{e}}^{j} .
\end{aligned}
$$

These equations contain the description of superconductors, magneto-hydrodynamic and the fluid-description of plasmas (e.g. see [8, 9]) as special cases. However, they are substantially more general due to the inclusion of the effect of entrainment, which is usually overlooked in these contexts.

Using the momenta (91) and the energy differential 90. the generalized pressure differential (40) is found as

$$
d \Psi=n d \mu+n_{\mathrm{e}} d \mu^{\mathrm{e}}-\alpha d \Delta^{2} .
$$

We note that in general we cannot introduce "partial pressures", say, by defining $d P_{e}$ to be equal $n_{\mathrm{e}} d \mu^{\mathrm{e}}$, as this is generally not a total differential due to interaction energies between the constituents (i.e. the fact that $\mu^{\mathrm{e}}=\mu^{\mathrm{e}}\left(n, n_{\mathrm{e}}, \boldsymbol{\Delta}^{2}\right)$ ). However, the chemical potentials are always well-defined and are therefore much more natural quantities in general multi-fluid contexts. In the absence of external forces, i.e. $f_{\text {ext }}=0$, the force balance equation (41) now reads as

$$
0=\boldsymbol{f}+\boldsymbol{f}^{\mathrm{e}}=\boldsymbol{f}_{\mathrm{H}}+\boldsymbol{f}_{\mathrm{H}}^{\mathrm{e}}-\sigma \boldsymbol{E}-\frac{\boldsymbol{j}}{c} \times \boldsymbol{B} .
$$

We can further prescribe a mutual force between the two fluids, so we introduce a resistivity force of the form $\boldsymbol{f}^{\mathrm{e}}=\boldsymbol{f}_{R}$, and therefore $f=-f_{R}$. The energy equation (30) with (24) now takes the form

$$
g+g^{\mathrm{e}}=-\boldsymbol{\Delta} \cdot \boldsymbol{f}_{R}=g_{\mathrm{ext}} .
$$

Such a resistive force will lead to creation of heat (entropy), which in this model has to be extracted to an "external" system via $g_{\text {ext }}$, as for simplicity we have not explicitly included an entropy constituent in this example. By the second law of thermodynamics, the friction should produce heat and not absorb it, so we have to extract heat-energy from the system, i.e. $g_{\text {ext }}<0$, and therefore we can constrain the resistivity force to be of the form

$$
\boldsymbol{f}_{R}=\eta \boldsymbol{\Delta}, \text { with } \eta>0,
$$

where $\eta$ is generally a function of the state-variables describing the system.

\section{B. The MHD limit}

In the low-frequency, long-wavelength limit we can assume any net charge densities to be compensated very quickly by the motion of electrons, so we make the "quasi-neutral" approximation and set:

$$
\sigma=0,
$$

which by (87) implies $Z n=n_{\mathrm{e}}$, and the current density therefore reads as

$$
\boldsymbol{j}=e n_{\mathrm{e}} \boldsymbol{\Delta} .
$$


In this low frequency limit we can equally neglect the displacement current $\partial_{t} \boldsymbol{D}$ in Maxwell's equations (18). Because the electrons are very light, i.e. $m_{\mathrm{e}} \ll m$, the inertial forces of the electron fluid can usually be neglected as well, and so the equation of motion for the electrons, $f^{\mathrm{e}}=\boldsymbol{f}_{R}$, can be written with (92), (94) and (98) as

$$
n_{\mathrm{e}} \nabla \mu^{\mathrm{e}}+2 \alpha \Delta_{j} \nabla v_{\mathrm{e}}^{j}+e n_{\mathrm{e}}\left(\boldsymbol{E}+\frac{\boldsymbol{v}_{\mathrm{e}}}{c} \times \boldsymbol{B}\right)=\eta \boldsymbol{\Delta}
$$

In order to recover the "standard" MHD framework, we further neglect entrainment, i.e. if we set $a d$ hoc $\alpha=0$, so the pressure differential (95) now reduces to

$$
d P=n_{\mathrm{e}} d \mu^{\mathrm{e}}+n d \mu
$$

where the generalized pressure $\Psi$ can be identified with the usual pressure $P$ in the absence of entrainment. This allows us to write the force balance equation (96) in the form

$$
\rho\left(\partial_{t}+\boldsymbol{v} \cdot \nabla\right) \boldsymbol{v}+\nabla P-\frac{\boldsymbol{j}}{c} \times \boldsymbol{B}=0
$$

Using (100) we can express the electron velocity as

$$
\boldsymbol{v}_{\mathrm{e}}=\boldsymbol{v}-\frac{1}{e n_{\mathrm{e}}} \boldsymbol{j},
$$

and so we can write the equation of electron transport (101) further as

$$
\boldsymbol{j}=\mathfrak{c}\left(\boldsymbol{E}+\frac{\boldsymbol{v}}{c} \times \boldsymbol{B}\right)+\frac{\mathfrak{c}}{e} \nabla \mu^{\mathrm{e}}-\frac{\mathfrak{c}}{e n_{\mathrm{e}} c} \boldsymbol{j} \times \boldsymbol{B}
$$

where the scalar conductivity $\mathfrak{c}$ is related to the resistivity coefficient $\eta$ as

$$
\mathfrak{c}=\frac{e^{2} n_{\mathrm{e}}^{2}}{\eta}>0 .
$$

If we further neglect the "partial pressure" $\nabla \mu$ e, we can write the relation between current $\boldsymbol{j}$ and electric field $\boldsymbol{E}^{\prime}$ in the frame of the ion-background, i.e. $\boldsymbol{E}^{\prime} \equiv \boldsymbol{E}+(\boldsymbol{v} / c) \times \boldsymbol{B}$ as a generalized Ohm's law, namely

$$
j_{i}=\mathfrak{c}_{i k} E^{k^{\prime}}
$$

where the anisotropic conductivity tensor $\mathfrak{c}_{i k}$ is

$$
\mathfrak{c}_{i k}=\left[\frac{1}{\mathfrak{c}} \delta_{i k}+\frac{1}{e n_{\mathrm{e}} c} \epsilon_{i k l} B^{l}\right]^{-1},
$$

which is not symmetric but satisfies the relation

$$
\mathfrak{c}_{i k}(\boldsymbol{B})=\mathfrak{c}_{k i}(-\boldsymbol{B}) .
$$

In this form the generalized Ohm's law can account for the well-known (classical) Hall effect, while the standard MHD approach (e.g. see [10,11]) commonly also neglects the "Hall term" $\boldsymbol{j} \times \boldsymbol{B}$, so that this equation finally reduces to the standard Ohm's law:

$$
\boldsymbol{j}=\mathfrak{c} \boldsymbol{E}^{\prime}=\mathfrak{c}\left(\boldsymbol{E}+\frac{1}{c} \boldsymbol{v} \times \boldsymbol{B}\right)
$$

We note that the "orthodox" equations are contained in this framework as special cases, but the description (101) is substantially more general due to the inclusion of the entrainment effect, which will generally be present in any (interacting) multi-fluid system.

\section{Superconductors}

In contrast to the previous application, superconductors are perfect conductors, so the electrons can flow past the ions without friction, i.e. $\eta=0$ in (98). Therefore we cannot neglect the inertial and pressure forces of the 
electrons a-priori. As mentioned previously (cf. Sect. IVB), in addition to the absence of friction, superfluids are also constrained to be irrotational, so

$$
\varpi_{i j}=\nabla_{[i} \pi_{j]}^{\mathrm{e}}=0
$$

and in its dual formulation this explicitly reads as

$$
\mathcal{W}^{\mathrm{e}}=\nabla \times \boldsymbol{\pi}^{\mathrm{e}}=\nabla \times \boldsymbol{p}^{\mathrm{e}}-\frac{e}{c} \boldsymbol{B}=0,
$$

which we will see after translation to the "orthodox" language represents the (second) London equation. In the absence of "external" forces acting on the electron fluid, using (23) we can reduce the equation of motion for the electrons, $f^{\mathrm{e}}=0$, to the form

$$
\begin{aligned}
0 & =\partial_{t} \boldsymbol{\pi}^{e}-\nabla \pi_{0}^{\mathrm{e}}=\partial_{t} \boldsymbol{p}^{\mathrm{e}}-\nabla p_{0}^{\mathrm{e}}+e\left(\nabla A_{0}-\frac{1}{c} \partial_{t} \boldsymbol{A}\right), \\
& =\partial_{t} \boldsymbol{p}^{\mathrm{e}}-\nabla p_{0}^{\mathrm{e}}+e \boldsymbol{E},
\end{aligned}
$$

where we have used (1516) and the definition (5) of the electric field $\boldsymbol{E}$. This equation is the (first) London equation and expresses the acceleration of electrons under gradients of their "potential" $p_{0}^{\mathrm{e}}$ and an electric field. This equation also guarantees that the constraint (112) remains automatically satisfied under the evolution of the electron fluid.

The two equations (112) and (113) were originally proposed (albeit in their "orthodox formulation") by F. and $\mathrm{H}$. London [12] and have been very successful in describing the phenomenology of superconductors, and in particular the behavior in electric and magnetic fields.

We conclude this section by a "translation" into the orthodox formalism (cf. the discussion of superfluid ${ }^{4} \mathrm{He}$ in Paper I). Using the entrainment relation (91) we can express the electron momentum $\boldsymbol{p}^{\mathrm{e}}$ as

$$
\frac{\boldsymbol{p}^{\mathrm{e}}}{m_{\mathrm{e}}}=\boldsymbol{v}-\frac{1}{e n_{\mathrm{S}}} \boldsymbol{j}
$$

where we have introduced the orthodox pseudo-density $n_{\mathrm{S}}$ of superconducting electrons, namely

$$
n_{\mathrm{S}} \equiv \frac{n_{\mathrm{e}}}{1-\varepsilon}, \quad \text { with } \quad \varepsilon \equiv \frac{2 \alpha}{m_{\mathrm{e}} n_{\mathrm{e}}} .
$$

With this relation, Eq. (112) can now be written in its conventional form as

$$
\boldsymbol{B}=-c \nabla \times(\lambda \boldsymbol{j}), \quad \text { with } \quad \lambda \equiv \frac{m_{\mathrm{e}}}{e^{2} n_{\mathrm{S}}},
$$

where we have assumed that the background of ions is stationary and non-rotating, so $\nabla \times \boldsymbol{v}=0$ and $\partial_{t} \boldsymbol{v}=0$. Therefore Eq. (113) can now be written as

$$
\boldsymbol{E}=\partial_{t}(\Lambda \boldsymbol{j})+\frac{1}{e} \nabla p_{0}^{\mathrm{e}}
$$

where the "partial pressure" term $\nabla p_{0}^{\mathrm{e}}=-\nabla\left(\mu^{\mathrm{e}}-\frac{1}{2} m_{\mathrm{e}} v_{\mathrm{e}}^{2}+\boldsymbol{v}_{\mathrm{e}} \cdot \boldsymbol{p}^{\mathrm{e}}\right)$ is often neglected. Eqs. (116) and (117) represent the orthodox formulation of the classic London equations as usually found in the superconductivity literature (e.g. see [13, 14, 15]).

\section{Acknowledgments}

I would like to thank Brandon Carter and David Langlois for many valuable discussions about the relativistic variational principle and superfluids. I am also very grateful to Greg Comer and Nils Andersson for many helpful comments.

I acknowledge support from the EU Programme 'Improving the Human Research Potential and the Socio-Economic Knowledge Base' (Research Training Network Contract HPRN-CT-2000-00137). 


\section{APPENDIX A: APPROXIMATE GALILEAN-INVARIANCE OF ELECTRODYNAMICS}

As pointed out in the introduction, the combined electro-hydrodynamics constructed in this paper makes no claim at being strictly Galilean-invariant. The underlying framework should be thought of as a fully (locally) Lorentzinvariant theory (as developed in 6, 7]), of which we consider only the small-velocity, low-frequency regime up to and including effects of order $\left(c^{-1}\right)$. It is well-known (e.g. see 16]) that the first post-Newtonian corrections to uncharged particle-mechanics in a gravitational field are of order $\left(c^{-2}\right)$, and the corresponding limiting theory is the strictly Galilean-invariant classical mechanics. This is not the case for electrodynamics. As pointed out in [5], one cannot obtain a Galilean-invariant limit and keep the full Maxwell-equations, except for introducing the infamous ether. However, as we will show here, by restricting ourselves to a suitable "non-relativistic" regime of small velocities and low frequencies, the full framework of electrodynamics admits an "approximately" Galilean invariant formulation up to and including order $\left(c^{-1}\right)$. This will still hold true for the combined electro-hydrodynamics, as there are no $\left(c^{-1}\right)$ effects entering from the mechanical sector of the theory. By consistently neglecting terms of order $\left(c^{-2}\right)$, the combined theory can be considered as "approximately" Galilean-invariant in this sense.

The well-known Lagrangian density $\Lambda_{\mathrm{EM}}$ of the electrodynamic field $F_{\mu \nu}=2 \nabla_{[\mu} A_{\nu]}$ can be equivalently expressed in terms of the two (frame-dependent) vectors $\boldsymbol{E}$ and $\boldsymbol{B}$, namely

$$
\Lambda_{\mathrm{EM}}=\frac{1}{16 \pi} F_{\mu \nu} F^{\nu \mu}=\frac{1}{8 \pi}\left(\boldsymbol{E}^{2}-\boldsymbol{B}^{2}\right),
$$

where $\boldsymbol{E}$ and $\boldsymbol{B}$, defined for an observer $u^{\mu}$, are given by

$$
E^{\mu}=F^{\mu \nu} u_{\nu}, \quad B^{\mu}=-\frac{1}{2} \epsilon^{\mu \nu \lambda \gamma} F_{\lambda \gamma} u_{\nu} .
$$

Note that both vectors are purely spatial and orthogonal to $u^{\mu}$. In the Lorentz-frame defined by $u^{\mu}$ they can therefore be identified with the common 3 -vectors used in the " $3+1$ " Newtonian language describing the electric and magnetic field. Conversely, the field-tensor $F_{\mu \nu}$ is uniquely specified in terms of $\boldsymbol{E}$ and $\boldsymbol{B}$, namely

$$
\underline{F}=\underline{u} \wedge \underline{E}+^{*}(\boldsymbol{u} \wedge \boldsymbol{B})
$$

where $*$ denotes the Hodge duality operation with respect to the 4-dimensional Levi-Civita tensor $\underline{\epsilon}$, and $\wedge$ stands for the exterior product. In the Lorentz-frame $u^{\mu}$ we can write this in components as

$$
F_{\mu \nu}=\left(\begin{array}{cccc}
0 & -E_{x} & -E_{y} & -E_{z} \\
E_{x} & 0 & B_{z} & -B_{y} \\
E_{y} & -B_{z} & 0 & B_{x} \\
E_{z} & B_{y} & -B_{x} & 0
\end{array}\right)
$$

Now consider the effect of the transformation to a frame $K^{\prime}$ moving with velocity $\boldsymbol{V}$ relative to the original frame $K$. Introducing the symbols $\boldsymbol{\beta} \equiv \boldsymbol{V} / c, \beta=|\boldsymbol{\beta}|, \boldsymbol{n}=\boldsymbol{\beta} / \beta$, and $\gamma=\left(1-\beta^{2}\right)^{-1 / 2}$, the corresponding Lorentztransformation matrix $\Lambda$ can be written as

$$
\begin{aligned}
& \Lambda_{0}^{0^{\prime}}=\gamma \\
& \Lambda^{0^{\prime}}{ }_{j}=\Lambda^{j^{\prime}}=-\beta \gamma n^{j}, \\
& \Lambda_{j}^{i^{\prime}{ }_{j}}=(\gamma-1) n^{j} n^{k}+\delta^{j k} .
\end{aligned}
$$

Using this, we can transform the frame-components of $F_{\mu \nu}$ to $K^{\prime}$ and translate them back into $\boldsymbol{E}^{\prime}$ and $\boldsymbol{B}^{\prime}$, which yields (e.g. see [16])

$$
\begin{array}{ll}
\boldsymbol{E}_{\|}^{\prime}=\boldsymbol{E}_{\|}, & \boldsymbol{E}_{\perp}^{\prime}=\gamma\left(\boldsymbol{E}_{\perp}+\boldsymbol{\beta} \times \boldsymbol{B}\right), \\
\boldsymbol{B}_{\|}^{\prime}=\boldsymbol{B}_{\|}, & \boldsymbol{B}_{\perp}^{\prime}=\gamma\left(\boldsymbol{B}_{\perp}-\boldsymbol{\beta} \times \boldsymbol{E}\right),
\end{array}
$$

where the parallel $(\|)$ and orthogonal $(\perp)$ projections refer to the boost-direction $\beta$.

The range of validity of the formalism developed in this paper is the "non-relativistic" small-velocity, low-frequency regime, so we introduce the small parameter $\varepsilon \ll 1$ and require that all characteristic velocities $\boldsymbol{v}$ satisfy $|\boldsymbol{v}| / c \sim(\varepsilon)$, and that time-derivatives of field-quantities are small compared to spatial derivatives, i.e. $\partial_{t} B / c \sim \partial_{t} E / c \sim(\varepsilon)$. Consequently we need to restrict ourselves to small Lorentz-boosts, i.e. we assume $\beta \sim(\varepsilon)$, and so

$$
\gamma=\left(1-\beta^{2}\right)^{-1 / 2}=1+\left(\varepsilon^{2}\right) .
$$


We can see that the Lorentz-transformation (A6A7) of the electromagnetic fields can now be written as

$$
\begin{aligned}
& \boldsymbol{E}^{\prime}=\boldsymbol{E}+\boldsymbol{\beta} \times \boldsymbol{B}+\left(\varepsilon^{2}\right), \\
& \boldsymbol{B}^{\prime}=\boldsymbol{B}-\boldsymbol{\beta} \times \boldsymbol{E}+\left(\varepsilon^{2}\right) .
\end{aligned}
$$

As pointed out in [5], these transformations do not form a strict Galilean invariance group, because they fail to be additive. But they do form an "approximate" invariance-group up to order $\left(\varepsilon^{2}\right)$ in the sense discussed above. Namely, combining two boosts, $\boldsymbol{\beta}_{1}$ and $\boldsymbol{\beta}_{2}$, we find

$$
\begin{aligned}
& \boldsymbol{E}^{\prime \prime}=\boldsymbol{E}^{\prime}+\boldsymbol{\beta}_{2} \times \boldsymbol{B}^{\prime}=\boldsymbol{E}+\left(\boldsymbol{\beta}_{1}+\boldsymbol{\beta}_{2}\right) \times \boldsymbol{B}+\left(\varepsilon^{2}\right), \\
& \boldsymbol{B}^{\prime \prime}=\boldsymbol{B}^{\prime}-\boldsymbol{\beta}_{2} \times \boldsymbol{E}^{\prime}=\boldsymbol{B}-\left(\boldsymbol{\beta}_{1}+\boldsymbol{\beta}_{2}\right) \times \boldsymbol{E}+\left(\varepsilon^{2}\right) .
\end{aligned}
$$

Let us consider the effect of the transformation A9 A10 on the electrodynamics field-Lagrangian (A1), for which we easily find

$$
\Lambda_{\mathrm{EM}}^{\prime}=\frac{1}{8 \pi}\left(\boldsymbol{E}^{\prime 2}-\boldsymbol{B}^{\prime 2}\right)=\frac{1}{8 \pi}\left(\boldsymbol{E}^{2}-\boldsymbol{B}^{2}\right)+\left(\varepsilon^{2}\right)
$$

In principle this would conclude our demonstration, as both the Maxwell-equations and the Lorentz-force law are derivable from this Lagrangian, but for completeness we will also discuss their explicit transformation properties.

Note that in the conventional Newtonian " $3+1$ " language, the boost $\boldsymbol{V}$ results in the following transformations (using the fact that $x^{0}=c t$ ) up to order $\left(\varepsilon^{2}\right)$ :

$$
\begin{aligned}
t^{\prime} & =t \\
\boldsymbol{x}^{\prime} & =\boldsymbol{x}-\boldsymbol{V} t \\
\boldsymbol{v}^{\prime} & =\boldsymbol{v}-\boldsymbol{V}, \\
\partial_{t}^{\prime} & \left.\equiv \partial_{t}\right|_{\boldsymbol{x}^{\prime}}=\partial_{t}+\boldsymbol{V} \cdot \nabla,
\end{aligned}
$$

which are just the usual Galilean boost transformations. Similarly, we obtain the transformation law up to $\left(\varepsilon^{2}\right)$ for the gauge-field vector $A_{\mu}=\left(A_{0}, \boldsymbol{A}\right)$ as

$$
\begin{aligned}
A_{0}{ }^{\prime} & =A_{0}+\boldsymbol{\beta} \cdot \boldsymbol{A}, \\
\boldsymbol{A}^{\prime} & =\boldsymbol{A}+A_{0} \boldsymbol{\beta},
\end{aligned}
$$

and one can easily verify using the definition (5) of $\boldsymbol{E}$ and $\boldsymbol{B}$ in terms of $\left(A_{0}, \boldsymbol{A}\right)$ that this is consistent with the transformations (A9A10) up to corrections of $\left(\varepsilon^{2}\right)$. It is also interesting to note the transformation properties of the charge-density $\sigma$ and the electrical current $j$. For an individual constituent $X$, the 4 -current $j_{X}^{\mu}=n_{X} u_{X}^{\mu}$, with the 4-velocity $u_{X}^{\mu}=\left(c, \boldsymbol{v}_{X}\right)+\left(\varepsilon^{2}\right)$. We therefore find up to order $\left(\varepsilon^{2}\right)$

$$
\sigma_{X}^{\prime}=\sigma_{X}, \quad \boldsymbol{j}_{X}^{\prime}=\boldsymbol{j}_{X}-\sigma_{X} \boldsymbol{V}
$$

and by the definition (3) of the total charge-density $\sigma$ and current $\boldsymbol{j}$ one easily finds ${ }^{3}$

$$
\sigma^{\prime}=\sigma, \quad \boldsymbol{j}^{\prime}=\boldsymbol{j}-\sigma \boldsymbol{V} .
$$

Applying the transformation (A9, A10) and (A14) to the Maxwell-equations (17), (18), we obtain

$$
\begin{aligned}
\nabla \cdot \boldsymbol{B}^{\prime} & =\boldsymbol{\beta} \cdot(\nabla \times \boldsymbol{E})=-\boldsymbol{\beta} \cdot\left(\partial_{t} \boldsymbol{B} / c\right)=\left(\varepsilon^{2}\right), \\
\nabla \times \boldsymbol{E}^{\prime}+\frac{1}{c} \partial_{t}^{\prime} \boldsymbol{B}^{\prime} & =\nabla \times \boldsymbol{E}+\frac{1}{c} \partial_{t} \boldsymbol{B}-\boldsymbol{\beta} \times \frac{1}{c} \partial_{t} \boldsymbol{E}-(\boldsymbol{\beta} \cdot \nabla)(\boldsymbol{\beta} \times \boldsymbol{E})+\left(\varepsilon^{2}\right) \\
& =\left[\nabla \times \boldsymbol{E}+\frac{1}{c} \partial_{t} \boldsymbol{B}\right]+\left(\varepsilon^{2}\right), \\
\nabla \cdot \boldsymbol{E}^{\prime}-4 \pi \sigma^{\prime} & =[\nabla \cdot \boldsymbol{E}-4 \pi \sigma]+\nabla \cdot(\boldsymbol{\beta} \times \boldsymbol{B})=-\boldsymbol{\beta} \cdot(\nabla \times \boldsymbol{B})=\left(\varepsilon^{2}\right),
\end{aligned}
$$

\footnotetext{
3 This shows that the current density always transforms according to the "electric limit" as defined in [5], irrespective of the presence of net charges. The authors overlooked the fact that even a space-like current has to be the sum of time-like "elementary" currents, and so the "magnetic limit" will never apply in real systems.
} 


$$
\begin{aligned}
\nabla \times \boldsymbol{B}^{\prime}-\frac{1}{c} \partial_{t}^{\prime} \boldsymbol{E}^{\prime}-\frac{4 \pi}{c} \boldsymbol{j}^{\prime}= & \nabla \times \boldsymbol{B}-\boldsymbol{\beta}(\nabla \cdot \boldsymbol{E})-\frac{1}{c} \partial_{t} \boldsymbol{E}-\boldsymbol{\beta} \times \frac{1}{c} \partial_{t} \boldsymbol{B}-(\boldsymbol{\beta} \cdot \nabla)(\boldsymbol{\beta} \times \boldsymbol{B}) \\
& -\left(\frac{4 \pi}{c} \boldsymbol{j}-4 \pi \sigma \boldsymbol{\beta}\right)+\left(\varepsilon^{2}\right) \\
= & {\left[\nabla \times \boldsymbol{B}-\frac{1}{c} \partial_{t} \boldsymbol{E}-\frac{4 \pi}{c} \boldsymbol{j}\right]+\left(\varepsilon^{2}\right), }
\end{aligned}
$$

where in the last equation we used the transformation property of the electrical current density $\boldsymbol{j}^{\prime}=\boldsymbol{j}-\sigma \boldsymbol{V}$.

Finally, the expression for the Lorentz-force (28) transforms as

$$
\frac{\boldsymbol{f}_{\mathrm{EM}}^{\prime}}{n q}=\boldsymbol{E}^{\prime}+\frac{\boldsymbol{v}^{\prime}}{c} \times \boldsymbol{B}^{\prime}=\boldsymbol{E}+\frac{\boldsymbol{v}}{c} \times \boldsymbol{B}-\frac{\boldsymbol{v}}{c} \times \boldsymbol{\beta} \times \boldsymbol{E}+\left(\varepsilon^{2}\right)=\frac{\boldsymbol{f}}{n q}+\left(\varepsilon^{2}\right),
$$

which concludes our demonstration of the approximate Galilean invariance of the equations.

[1] R. Prix, Phys. Rev. D. 69, 043001 (2004).

[2] B. Carter and N. Chamel, Int. J. Mod. Phys D13, 291 (2004), preprint: astro-ph/0305186.

[3] B. Carter and N. Chamel, astro-ph/0312414 (2003).

[4] B. Carter and N. Chamel, astro-ph/0410660 (2004).

[5] M. Le Bellac and J.-M. Lévy-Leblond, Nuovo Cimento 14, 217 (1973).

[6] B. Carter and D. Langlois, Nucl. Phys. B531, 478 (1998).

[7] B. Carter, in Vortices in Unconventional Superconductors and Superfluids, edited by R. P. Huebener, N. Schopohl, and G. Volovik (Springer Verlag, 2002, (preprint: astro-ph/0010109)).

[8] S. Chandrasekhar, Plasma Physics (Phoenix books. The University of Chicago Press, 1960).

[9] J. P. Freidberg, Ideal Magnetohydrodynamics (Plenum Press, New York, 1987).

[10] T. G. Cowling, Magnetohydrodynamics, Monographs on Astronomical Subjects (Adam Hilger, England, 1976).

[11] J. D. Jackson, Classical Electrodynamics (John Wiley \& Sons, Inc., 1975).

[12] F. and H. London, Proc. Roy. Soc. 149, 71 (1935).

[13] F. London, Superfluids. Vol. I. Macroscopic Theory of Superconductivity (Wiley, New York, 1950).

[14] D. R. Tilley and J. Tilley, Superfluidity and Superconductivity (IOP Publishing, 1990).

[15] M. Tinkham, Introduction to superconductivity (McGraw-Hill, 1996), 2nd ed.

[16] C. W. Misner, K. S. Thorne, and J. A. Wheeler, Gravitation (W. H. Freeman and Company (New York), 1973). 